\section{Activated protein C anticoagulant activity is enhanced by skeletal muscle myosin}

Skeletal muscle myosin (SkM) promotes thrombus formation in flowing blood, supports prothrombinase activity in plasma, and promotes prothrombin activation by factors (F) Xa and Va in purified systems. ${ }^{1}$ SkM binds FXa and FVa and effectively replaces the procoagulant activity of phospholipid vesicles. This discovery challenges a long-standing paradigm for blood coagulation in which coagulation reactions, except for contact activation reactions, are thought to occur only on surfaces containing negatively charged phospholipids, which include phosphatidylserine..$^{2-6}$ Thrombin provides both positive feedback upregulation of coagulation by activating various clotting factors and negative feedback downregulation of coagulation by activating protein $\mathrm{C}$ to the potent anticoagulant, activated protein $\mathrm{C}$ (APC). ${ }^{2-6}$ Because APC is potent for negative feedback downregulation of thrombin generation in vivo, ${ }^{7}$ here we assessed and describe that SkM enhances the anticoagulant activity of APC in purified human clotting factor reaction mixtures and in human plasma. This indicates that SkM can promote both thrombin generation and negative feedback downregulation of thrombin generation.

Because the major mechanism of the anticoagulant activity of APC involves irreversible proteolytic inactivation of FVa, purified human FVa was incubated with APC and varying concentrations of SkM; then residual FVa activity was determined (see the Online Supplementary Appendix for details of the methods and the reagents used). These SkM dose-response studies for FVa inactivation by APC showed a maximum effect at SkM concentrations of 30-40 $\mathrm{nM}$ and a half-maximal effect (EC50) at $10 \mathrm{nM}$ SkM (Figure 1A). When the time-course for FVa inactivation by APC in the presence of $40 \mathrm{nM} \mathrm{SkM}$ was monitored and compared to that in the presence of $25 \mu \mathrm{M}$ phospholipids, the rate and extent of FVa inactivation by APC were very similar for SkM and for phospholipids (Figure 1B). To evaluate whether phosphatidylserine contamination of SkM might be responsible for the activity of SkM, the purified SkM reagent was submitted to Avanti Polar Lipids, Inc. (Alabaster, AL, USA) and analyzed using liquid chromatography mass spectrometry. This analysis showed that $40 \mathrm{nM}$ SkM contained only 1.0 $\mathrm{nM}$ phosphatidylserine which could not possibly explain why $40 \mathrm{nM} \mathrm{SkM}$ is as potent as the optimal level of $25 \mu \mathrm{M}$ phospholipids. Notably, in separate studies, 40 $\mathrm{nM}$ phospholipids provided negligible inactivation of $\mathrm{FVa}$ (data not shown).

Because protein $S$ is an anticoagulant cofactor for APC, the ability of protein $S$ to enhance SkM-dependent inactivation of FVa by APC was assessed. When the time course for FVa inactivation by APC in the absence and presence of protein $\mathrm{S}$ was determined, protein $\mathrm{S}$ significantly enhanced this reaction (Figure $1 \mathrm{C}$ ). The initial rate of FVa inactivation by APC was maximally enhanced four-fold by the optimal level of $100 \mathrm{nM}$ protein $\mathrm{S}$ (Figure 1C). The effects of SkM on specific proteolytic cleavages of FVa by APC in the absence and presence of protein $S$ were examined by immunoblotting using a monoclonal antibody that is directed against an epitope in the 307506 amino acid region of the FVa heavy chain (Figure 1D). Bands were identified by epitope, by apparent molecular mass, and by comparison to bands previously identified in studies of FVa and of FVa cleavage site mutants. ${ }^{8,9}$ As seen in the immunoblot (Figure 1D), the most visually prominent FVa heavy chain fragment due to cleavage by APC in the presence of SkM but absence of protein $S$ was due to cleavage at Arg506, resulting in appearance of the 1-506 amino acid fragment. But strikingly, when protein $S$ was present, the visually evident cleavage by APC in the presence of SkM was at Arg306 because the most prominent fragment was the 307-506 amino acid fragment. Such SkM-supported cleavages are consistent with previous studies which showed that, in the presence of phospholipids, protein $S$ enhances the cleavage of APC at R306. ${ }^{10,11}$ Thus, SkM may function as an anticoagulant APC cofactor.

To determine whether SkM enhances APC anticoagulant activity in the milieu of plasma, clotting assays were employed using protocols that avoided adding exogenous phospholipid vesicles which themselves would promote the anticoagulant effects of APC. When the effects of varying levels of SkM on FXa one-stage assays were studied, there was a procoagulant effect of increasing SkM concentrations in the absence of addition of APC, i.e., there was a shortening of the clotting times from 87 to $52 \mathrm{~s}$ (Figure 1E), consistent with the known procoagulant effects of SkM in plasma. ${ }^{1}$ Strikingly, in the presence of APC, SkM addition increased rather than decreased the clotting times, e.g., from 129 to 207 s (Figure 1E), showing that SkM enhanced the anticoagulant actions of $\mathrm{APC}$ in plasma. The APC sensitivity ratio, defined here as the ratio of clotting times in the presence of APC compared to in its absence, rose significantly with increasing SkM concentrations from 1.5 in the absence of SkM to 3.9 at $200 \mathrm{nM} \mathrm{SkM} \mathrm{(Figure} \mathrm{1E).} \mathrm{Kaolin-induced} \mathrm{clotting}$ time assays were also used with plasma conditions chosen to emphasize that changes were due to FVa inactivation in plasma. For this purpose, when FV-deficient plasma was reconstituted with $2 \mathrm{nM}$ of $\mathrm{FVa}$, addition of increasing concentrations of SkM produced significant procoagulant effects as clotting times were reduced from 126 to $71 \mathrm{~s}$ (Figure 1F). However, when APC was present, addition of SkM produced significant anticoagulant effects as clotting times were prolonged from 187 to 306 $s$ (Figure 1F). The calculated APC sensitivity ratio rose significantly, as a function of increasing SkM concentrations, from 1.4 to 4.2 (Figure 1F). Thus, data here from two different coagulation assays unambiguously demonstrate that SkM can enhance the anticoagulant actions of APC, not only in purified reaction mixtures but also in the plasma environment.

SkM can provide a surface on which both procoagulant and anticoagulant reactions, namely prothrombin activation 1 and FVa inactivation, respectively, are enhanced. So here we tested the ability of SkM to provide a surface for enhancement of protein $\mathrm{C}$ activation by thrombin or thrombin:thrombomodulin, and we found no influence of SkM on protein C activation. When SkM was tested at concentrations ranging from 5 to $100 \mathrm{nM}$, it did not change the rate of protein $\mathrm{C}$ activation by thrombin in purified reaction mixtures with or without calcium ions (Figure 2A), by thrombin and soluble thrombomodulin in the presence of calcium ions (Figure 2B), or by thrombin in the presence of endothelial cells (Figure 2C). Thus, SkM had no effect on protein $\mathrm{C}$ activation. The fact that SkM does not support the activation of protein $C$ by thrombin \pm thrombomodulin in purified reaction mixtures or on endothelial cells suggests that SkM enhancement of protein $C$ activation is unlikely to occur in vivo, although this idea needs in vivo experimentation in order that a firm conclusion can be reached.

Since procoagulant and anticoagulant mechanisms must normally be balanced overall in vivo and since phospholipids enhance both thrombin generation and APC 
anticoagulant activity, here we investigated whether SkM could support not only procoagulant prothrombinase reactions but also the balancing anticoagulant activities by APC and protein S. In purified clotting factor mixtures, SkM enhanced APC/protein S proteolytic inactivation of FVa. In two distinct types of plasma clotting assays, kaolin-induced clotting time and FXa one-stage assays, SkM dose-dependently shortened plasma clotting times in the absence of APC by enhancing prothrombinase activity; however, in the presence of APC, SkM strikingly prolonged clotting times, implying that $\mathrm{SkM}$ is an anticoagulant APC cofactor. Thus, these studies extend the potential role of SkM in supporting procoagulant reactions ${ }^{1}$ to that of supporting the anticoagulant actions of APC and protein S, indicating that SkM may contribute to negative feedback downregulation of thrombin generation (Figure 3)

The possibility that SkM might contribute to traumainduced coagulopathy was suggested by the observation that SkM is increased in plasma of trauma patients ${ }^{1}$ and
A

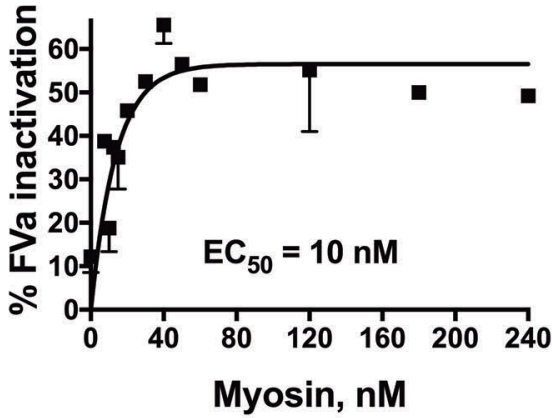

C

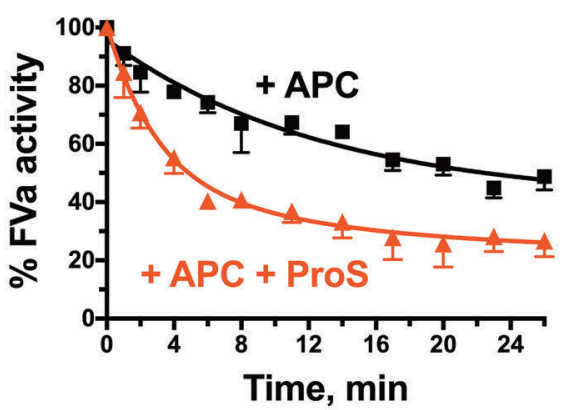

E

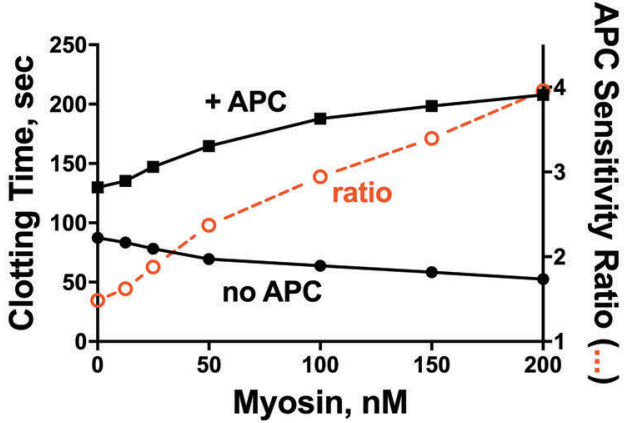

B

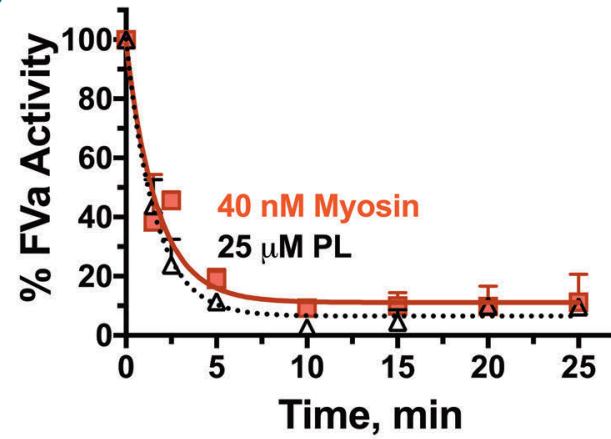

D

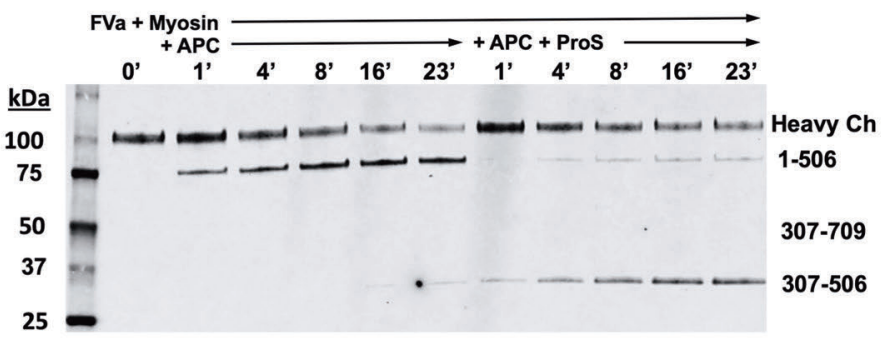

$\mathbf{F}$

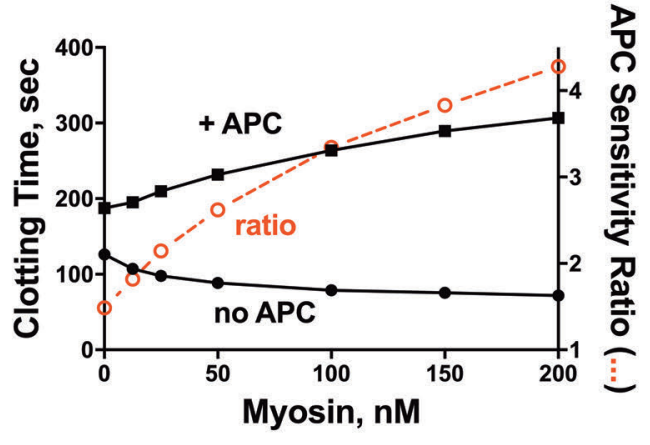

Figure 1. Myosin supports inactivation of activated factor $\mathrm{V}$ by activated protein $\mathrm{C}$ in purified human coagulation factor reaction mixtures and in human plasma coagulation assays. (A) Skeletal muscle myosin (myosin) dose-response curve for inactivation of $1.25 \mathrm{nM}$ activated factor $\mathrm{V}$ (FVa) by $0.4 \mathrm{nM}$ activated protein $\mathrm{C}$ (APC) over 4 min. Data shown are the composite from three experiments performed on different days. (B) Time course for inactivation of $1 \mathrm{nM} F \mathrm{Fa}$ by $0.4 \mathrm{nM}$ $\mathrm{APC}$ in the presence of $40 \mathrm{nM}$ myosin, or $25 \mu \mathrm{M}$ phospholipids (PL). Residual FVa activity was measured in a second-stage standard prothrombinase assay with $\mathrm{PL}$, activated factor X (FXa) and prothrombin. One hundred percent FVa activity corresponded to generation of $\sim 1 \mathrm{nM}$ thrombin/min from the sub-aliquot used to test for prothrombinase activity in the absence of APC in the second stage of the assay. Data shown are the composite from three or four experiments performed on different days. (C) Inactivation of FVa (3.7 nM final concentration) by APC (0.6 nM final concentration) with or without protein S (100 nM final concentration), as indicated, in the presence of $120 \mathrm{nM}$ myosin. Data shown are combined from experiments performed on three different days. (D) Immunoblot analysis of reaction mixtures from FVa inactivation studies. Inactivation of FVa (3.7 nM final concentration) by APC ( $1 \mathrm{nM}$ final concentration) with or without protein S (100 nM final concentration) in the presence of $120 \mathrm{nM}$ myosin was monitored at the indicated times. Aliquots were taken over time into $80^{\circ} \mathrm{C}$ LDS Li-Cor sample buffer containing $10 \mathrm{mM}$ EDTA for $10 \mathrm{~min}$ for immunoblotting in sodium dodecylsulfate polyacrylamide gel electrophoresis. Immunoblotting of FVa was performed using a monoclonal anti-FVa heavy chain antibody with an epitope in the region of residues 307-506. (E) Factor Xa one-stage assay in FX-depleted plasma with FXa added shows the effects of myosin on clotting times in the presence of $17 \mathrm{nM}$ APC $(+$ APC) $(\bullet)$ and in the absence of added APC (no APC) ( $\bullet$ ). The APC sensitivity ratio $(O)$ was obtained by dividing the clotting times in the presence of APC by the clotting times in the absence of APC at each myosin concentration. (F) Kaolin clotting time assays in FV-deficient plasma with FVa added show the effects of myosin on clotting times in the presence of 17 nM APC (+ APC) ( $\mathbf{m})$ and in the absence of added APC (no APC) (•). The APC sensitivity ratio was calculated as in (E). 
that anti-SkM antibodies can significantly reduce thrombin generation in plasma from patients with traumainduced coagulopathy. ${ }^{1}$ The novel APC cofactor activity of SkM discovered here may have direct relevance for trauma-induced coagulopathy. One major mechanism thought to contribute to this form of coagulopathy involves hyperactivation of the protein $\mathrm{C}$ system, with consumption of FV and FVIII, and hyperfibrinolysis. ${ }^{12-15}$ It has been noted that $25-33 \%$ of severely injured patients present with elevated APC plasma levels, which are associated with increased blood transfusion requirements and mortality. ${ }^{15}$ This so-called "activated protein C traumainduced coagulopathy mechanism" is linked to suppres- sion of coagulation and enhanced fibrinolysis. ${ }^{15}$ Our discovery that SkM has APC cofactor activity raises a number of new questions about the mechanisms of traumainduced coagulopathy, such as whether trauma-induced exposure of SkM can causally contribute to excessive suppression of coagulation and hemostasis in subsets of trauma patients with increased bleeding risk. Further studies of SkM levels and activities in patients with trauma-induced coagulopathy and in preclinical trauma models are needed. Coagulation studies in the future will need to take these newly revealed mechanisms for diverse activities of SkM (Figure 3) into account and determine the potential physiological relevance for the
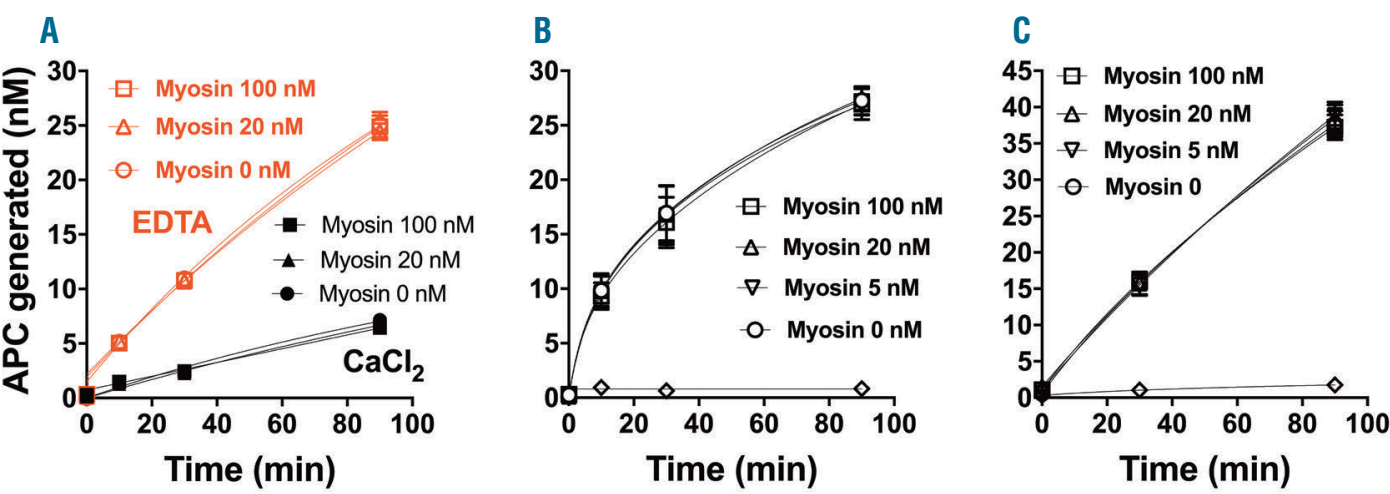

Figure 2. Myosin does not alter activation of protein $\mathrm{C}$ by thrombin or thrombin:thrombomodulin. (A) Protein $\mathrm{C}$ activation in purified component reaction mixtures by thrombin without thrombomodulin. Time course for protein $\mathrm{C}$ activation $(500 \mathrm{nM})$ by thrombin $(50 \mathrm{nM})$ in the absence of soluble thrombomodulin and in the presence or absence of various indicated concentrations of skeletal muscle myosin (myosin), with either 2 mM EDTA (red symbols) or 3 mM CaCl 2 (black symbols) at $37^{\circ} \mathrm{C}$. The diluent was Hepes buffer saline $+0.1 \%$ bovine serum albumin. (B) Protein $\mathrm{C}$ activation by thrombin with soluble thrombomodulin. Time course for protein $\mathrm{C}$ activation in a purified component system containing soluble thrombomodulin ( $5 \mathrm{nM})$ in the presence or absence of various indicated concentrations of myosin and the same general conditions as in (A) with $\mathrm{CaCl}_{2}$. Samples were collected at several times after the addition of $10 \mathrm{nM}$ thrombin and assayed for activated protein C (APC) amidolytic activity. Open diamonds indicate no thrombin added control. (C) Protein $\mathrm{C}$ activation by thrombin in the presence of endothelial cells. Time course for protein $\mathrm{C}$ activation on cells in the presence or absence of myosin. Thrombin (10 nM), protein C (100 nM) and various concentrations of myosin in Hank balanced salt solution $+1.3 \mathrm{mM} \mathrm{CaCl} 2+0.6 \mathrm{mM} \mathrm{MgCl}_{2}+0.1 \%$ endotoxin-free bovine serum albumin were added on confluent monolayers of EA.hy 926 cells. Samples of the fluid phase were collected 30 and 90 min after addition of thrombin and assayed for APC amidolytic activity. Open diamonds indicate no thrombin added control. Each point in (A), (B), and (C) represents the mean \pm standard error mean from three independent experiments.

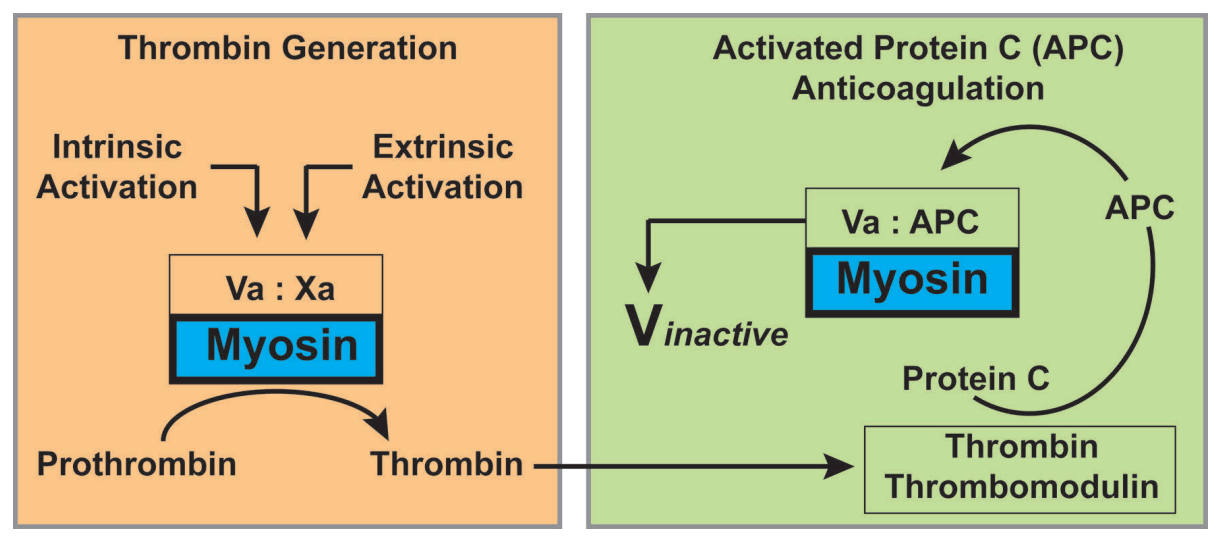

Figure 3. Skeletal muscle myosin can play a balancing role involving key blood coagulation reactions. In the left panel, factor Xa and its potent cofactor, factor Va, assemble to form the prothrombinase complex on surfaces such as skeletal muscle myosin (as shown here) or alternatively on the widely recognized procoagulant surface of negatively charged phospholipid membranes (not depicted here). The prothrombinase complex generates thrombin from prothrombin. In the right panel, some of the thrombin formed by the prothrombinase complex binds to thrombomodulin on cell surfaces and generates anticoagulant activated protein C (APC) from protein C. Then, in the presence of myosin, as depicted (or negatively charged phospholipid membranes, not depicted), APC can proteolytically inactivate factor Va, resulting in less thrombin generation. Thus, myosin may not only help to generate thrombin but may also contribute to negative feedback downregulation of excessive thrombin generation. During acute muscle trauma, blood is exposed to myosin and some myosin is released and circulates in plasma. The procoagulant role of skeletal muscle myosin as a membrane substitute could be important in situations such as acute muscle trauma to stop excessive bleeding. The anticoagulant role of skeletal muscle myosin could be important after initial trauma and tissue damage to help reduce excessive generation of thrombin and procoagulant factors. 
procoagulant and anticoagulant properties of SkM, especially in the context of trauma-induced coagulopathy.

Mary J. Heeb, José A. Fernández, Atsuki Yamashita, Olivia R. McDowell, Zihan Guo, Laurent O. Mosnier, Hiroshi Deguchi and John H. Griffin

Department of Molecular Medicine, The Scripps Research Institute, La Jolla, CA, USA

Correspondence: MARY J. HEEB/JOHN H. GRIFFIN

heeb@scripps.edu/igriffin@scripps.edu

doi:10.3324/haematol.2019.242982

Information on authorship, contributions, and financial \& other disclosures was provided by the authors and is available with the online version of this article at $W$ WW. haematologica.org.

\section{References}

1. Deguchi H, Sinha RK, Marchese P, et al. Prothrombotic skeletal muscle myosin directly enhances prothrombin activation by binding factors Xa and Va. Blood. 2016;128(14):1870-1878.

2. Furie B, Furie BC. Mechanisms of thrombus formation. New Eng J Med. 2008;359(9):938-949.

3. Versteeg HH, Heemskerk JW, Levi M, Reitsma PH. New fundamentals in hemostasis. Physiol Rev. 2013;93(1):327-358

4. Bevers EM, Williamson PL. Getting to the outer leaflet: physiology of phosphatidylserine exposure at the plasma membrane. Physiol Rev. 2016;96(2):605-645.

5. Flaumenhaft R. Thrombus formation reimagined. Blood. 2014; 124(11):1697-1698

6. Ivanciu L, Krishnaswamy S, Camire RM. New insights into the spa- tiotemporal localization of prothrombinase in vivo. Blood. 2014; 124(11):1705-1714

7. Gresele P, Momi S, Berrettini M, et al. Activated human protein C prevents thrombin-induced thromboembolism in mice. Evidence that activated protein $\mathrm{C}$ reduces intravascular fibrin accumulation through the inhibition of additional thrombin generation. J Clin Invest. 1998;101(3):667-676.

8. Heeb MJ, Rehemtulla A, Moussalli M, Kojima Y, Kaufman RJ. Importance of individual activated protein $C$ cleavage site regions in coagulation factor $\mathrm{V}$ for factor $\mathrm{Va}$ inactivation and for factor Xa activation. Eur J Biochem. 1999;260(1):64-75

9. Hockin MF, Cawthern KM, Kalafatis M, Mann KG. A model describing the inactivation of factor Va by APC: bond cleavage, fragment dissociation, and product inhibition. Biochemistry. 1999;38(21):69186934.

10. Rosing J, Hoekema L, Nicolaes GA, et al. Effects of protein S and factor Xa on peptide bond cleavages during inactivation of factor $V a$ and factor VaR506Q by activated protein C. J Biol Chem. 1995; 270(46):27852-27858

11. Kalafatis M, Mann KG. Role of the membrane in the inactivation of factor Va by activated protein C. J Biol Chem. 1993;268(36):2724627257.

12. Brohi K, Cohen MJ, Ganter MT, Matthay MA, Mackersie RC, Pittet JF. Acute traumatic coagulopathy: initiated by hypoperfusion: modulated through the protein C pathway? Ann Surg. 2007;245(5):812818.

13. Cohen MJ, Brohi K, Ganter MT, Manley GT, Mackersie RC, Pittet JF. Early coagulopathy after traumatic brain injury: the role of hypoperfusion and the protein C pathway. J Trauma. 2007;63(6):1254-1261.

14. Cohen MJ, Call M, Nelson M, et al. Critical role of activated protein $\mathrm{C}$ in early coagulopathy and later organ failure, infection and death in trauma patients. Ann Surg. 2012;255(2):379-385.

15. Kornblith LZ, Moore HB, Cohen MJ. Trauma-induced coagulopathy: the past, present, and future. J Thromb Haemost. 2019;17(6):852862. 